\title{
GC/MS Monitoring of Selected PAHs in Soil Samples Using Ultrasound-assisted QuEChERS in Tandem with Dispersive Liquid-Liquid Microextraction
}

\author{
Hamid Reza Sobhi \\ Department of Chemistry, Payame Noor University, Tehran, Iran
}

Email address:

hrsobhi@gmail.com

\section{To cite this article:}

Hamid Reza Sobhi: GC/MS Monitoring of Selected PAHs in Soil Samples Using Ultrasound-assisted QuEChERS in Tandem with Dispersive Liquid-Liquid Microextraction. International Journal of Environmental Monitoring and Analysis. Vol. 3, No. 5, 2015 , pp. $288-292$. doi: 10.11648/j.ijema.20150305.17

\begin{abstract}
Herein, a method consisting of ultrasound-assisted QuEChERS in tandem with dispersive liquid-liquid microextraction (DLLME) was developed for monitoring of selected polycyclic aromatic hydrocarbons (PAHs) in various soil samples using gas chromatography coupled to a single quadrupole mass spectrometry (GC/MS). Ultrasound-assisted QuEChERS was employed to extract the PAHs from $2.0 \mathrm{~g}$ of soil using $7 \mathrm{ml}$ of initial extraction solvent (acetonitrile: water $(5: 2 \mathrm{v} / \mathrm{v}))$ and the salt mixture. The resulting supernatant extract was cleaned through the addition of $\mathrm{C}_{18}$, PSA and the mix salt followed by centrifugation, decantation and filtration. Of the clean organic phase, $1.0 \mathrm{ml}$ was withdrawn and added with12 $\mu 1$ of $\mathrm{C}_{2} \mathrm{Cl}_{4}$ (disperser solvent). The resulting mixture was then injected rapidly into an aqueous sample $(5.0 \mathrm{ml})$ by a syringe for further preconcentration. As a result, the cloudy solution consisting of fine particles of the extraction solvent dispersed into the aqueous phase was formed. After centrifuging, the fine particles were sedimented at the bottom of the conical test tube $(5.0 \pm$ $0.5 \mu 1)$. Of which, $1.0 \mu \mathrm{l}$ was injected to the GC/MS for monitoring of the PAHs. Several influential parameters including ultrasound extraction time, initial extraction and disperser solvent and their respective volumes were all evaluated to achieve the optimal conditions. Under the optimal conditions, limits of quantification (2.5-4.0 ng/g) and linear ranges ( $\left.\mathrm{r}^{2} \geq 0.98\right)$ were obtained for the PAHs. The method was then successfully applied for the extraction and monitoring of the PAHs in the real soil samples. Accuracy of the method was evaluated by the relative recovery experiments on spiked samples with the results ranging from 81 to $92 \%$. In the mean time, the relative standard deviations (RSDs) were found to be in the range of $4.8-15.9 \%$.
\end{abstract}

Keywords: PAHs, Ultrasound-Assisted QuEChERS, Dispersive Liquid-Liquid Microextraction, Soil Samples

\section{Introduction}

Polycyclic aromatic hydrocarbons (PAHs) are found in environmental depositions, asa result of environmental pollution (e.g. oil spills) and can arise due to human activity (e.g. traffic, incineration, etc.) [1]. These pollutants have a high persistence in the environment, low bio-degradability and high lipophilicity and toxicicity [2-4].

Soil is a complex and heterogeneous matrix, containing both inorganic and organic components [5] and is often subject tointense chemical pollution. When chemical compounds reachthe soil, either via direct intentional application or as a result of accidental spillage [6, 7].

The amount of PAHs in soils is of importance because of their toxicity to humans andtheir effects on soil organisms and plants[8].As a result of theirwidespread presence, PAHs are generally introduced in monitoringprograms [9] and are listed by the US Environmental ProtectionAgency (EPA) and the European Community as priority pollutants[10].

Due to the low concentration levels of soil pollutants, sample preparation step is needed to determine the type and quantity of pollutant present [11] and to avoid interferences and improve thesensitivity of the method. To extract contaminants from soil, atechnique strong enough to extract bound residues is necessary [12].

Amongst the methods commonly applied for the extraction of pollutants from soil, QuEChERS is veryflexible, modifiable, and is growing in popularity due to all thebenefits described by its name: Quick, Easy, Cheap, Effective, Rugged and Safe. However, its effectiveness is dependent onthe analyte properties, matrix composition, equipment, andanalytical 
technique available in the laboratory [13-15].

Although it is possible to detect PAHs at low levels by GC/MS analysis which follows QuEChERS method; however, the use of another extraction procedure for better clean-up and further preconcentration is needed to reduce the effects of soil's matrix as much as possible. For that reason, in this study a highly efficient preconcentration procedure known as dispersive liquid-liquid microextraction (DLLME) along with QuEChERS was employed [16-18].

The present study outlines a novel modified method in which the initial extraction of selected PAHs was carried out by ultrasound assisted QuEChERS treatment followed by a further preconcentration by DLLME procedure. Our aim was focused at studying the feasibility of DLLME procedure for further preconcentration along with ultrasound assisted QuEChERS treatment in soil's PAHs analysis. To the best of our knowledge, so far this has been the first report on the extraction and measurement of PAHs in soil samples based on the combination of ultrasound assisted QuEChERS treatment and DLLME procedure.

\section{Instruments}

The gas chromatographic system consisted of an Agilent (Centerville Road, Wilmington, USA) series 7890A GC coupled to an Agilent MSD 5975C quadrupole mass spectrometer. The GC was fitted with HP-5 MS capillary column (30 $\mathrm{m} \times 0.25 \mathrm{~mm}$ i.d., $0.25-\mu \mathrm{m}$ film thickness) from Agilent J\&W Scientific (Folsom, CA, USA). Helium $(99.999 \%)$ was used as the carrier gas at the flow rate of 1.0 $\mathrm{ml} / \mathrm{min}$. The following temperature program was employed for the separation: $80{ }^{\circ} \mathrm{C}$ for $1 \mathrm{~min}$, increased to $280{ }^{\circ} \mathrm{C}$ at $8{ }^{\circ} \mathrm{C} / \mathrm{min}$, and held for $6 \mathrm{~min}$; finally increased to $300^{\circ} \mathrm{C}$ at $50{ }^{\circ} \mathrm{C} / \mathrm{min}$ and held for $3 \mathrm{~min}$. The MS quadrupole and the MS source temperatures were set at 150 and $230{ }^{\circ} \mathrm{C}$, respectively. Data acquisition was performed in the full scan mode $(\mathrm{m} / \mathrm{z}$ in the range of $50-400)$ to confirm the retention times of analytes and in selected ion monitoring (SIM) mode (see Table 1) for quantitative determination of PAHs. A dwell time of 100 ms was used for each mass operated at SIM mode with high resolution. The filament delay time was set at $3 \mathrm{~min}$. A vortexhomogenizer was applied to homogenize both the blank and real dried soil samples prior to each extraction. An ultrasound probe (UP-500 ultrasound homogenizer) from ECHROM Company (Avagene, Taiwan) was used for the extraction of the test PAHs from dried soil matrices.

\section{Exprimental}

\subsection{Samples and Reagents}

The test PAHs (acenaphthylene, acenaphthene, fluorene, phenanthrene, anthracene, fluoranthene, pyrene and chrysene) was purchased from Aldrich (Milwaukee, WI, USA). Analytical grade acetonitril $(\mathrm{ACN})$, tetrachlroethylene $\left(\mathrm{C}_{2} \mathrm{Cl}_{4}\right)$, Acetone, anhydride magnesium sulfate $\left(\mathrm{MgSO}_{4}\right)$ and sodium chloride $(\mathrm{NaCl})$ were supplied by Merck Company (Darmstadt,
Germany). Primary secondary amine (PSA) and octadecylsilane $\left(\mathrm{C}_{18}\right)$ sorbents were obtained from Varian (Varian, Harbor City, CA). Pure water was provided by a home-made deionizer. Stock standard solutions $(500 \mathrm{mg} / \mathrm{L})$ of PAHs were prepared in acetonitrile and then they were diluted with acetone (as working solutions) and then all stored at $20^{\circ} \mathrm{C}$. A number of soil samples were collected twice a week for one month in order to obtain the representative samples over this period of time. Upon their arrival at the laboratory, the samples were dried and sieved in order to obtain a fraction of $<1 \mathrm{~mm}$ and maintained at $4^{\circ} \mathrm{C}$ until the time of analysis. For constructing the calibration curve, ten standard solutions in the range of $2.0-100 \mathrm{ng} / \mathrm{g}$ were prepared by spiking the working solution intothe blank samples- later confirmed to be the PAHs free. The standard solutions were stored at $-20^{\circ} \mathrm{C}$ until final analysis. Meanwhile, the suspected soil samples (hereafter, called real samples) were taken from three different parts of Tehran (Tehran, Iran).

\subsection{Sample Preparation}

\subsubsection{Extraction Procedure}

1) $2.0 \mathrm{~g}$ of the treated soil sample (already dried, filtered and spiked/non-spiked with the PAHs) was placedin a $50-\mathrm{mL}$ ultrasound probe.

2) $7.0 \mathrm{~mL}$ of $\mathrm{ACN}$ :Water $(5: 2, \mathrm{v} / \mathrm{v})$ was added to the probeandit was vibrated using ultrasound energy for $3 \mathrm{~min}$.

3) The mix salt $\left(\mathrm{MgSO}_{4}: \mathrm{NaCl} ; 4: 1 \mathrm{~g}\right)$ was added to the above solution.

4) The resulting mixture was immediately shaken vigorously for $2 \mathrm{~min}$ and centrifuged at $3000 \mathrm{rpm}$ for $5 \mathrm{~min}$.

\subsubsection{Clean-Up}

5) The upper organic phase was removed and transferred to an Eppendorf vial. Then it was added with 50, 50 and 150 $\mathrm{mg}$ of $\mathrm{C}_{18}$, PSA and $\mathrm{MgSO}_{4}$, respectively.

6) The final extract (the supernatant) was filtered through a $0.22 \mu \mathrm{m}$ PTFE syringe filter to obtain $\sim 1 \mathrm{ml}$ of ACN.

\subsubsection{Preconcentration}

7) $12 \mu \mathrm{l}$ of tetrachloroethylene $\left(\mathrm{C}_{2} \mathrm{Cl}_{4}\right)$ was added to the $\mathrm{ACN}$ and the resulting mixture was rapidly injected to a 5.0 $\mathrm{ml}$ of pure water for further preconcentration.

8) The sedimented organic phase $(5 \pm 0.5 \mu \mathrm{l})$ was immediately formed and withdrawn. Of which, $1.0 \mu \mathrm{l}$ was injected to a GC/MS for identification and measurement of the PAHs.

\section{Evaluation of Analytical Parameters}

The evaluated analytical parameters include: Selectivity, relative recovery, precision (RSD), linearity and limit of quantification (LOQ). They were all evaluated for each PAH using spiked and non-spiked blank soil samples.

\subsection{Selectivity}

The selectivity of the method was demonstrated by injecting the diluted working solution of the test PAHs. In addition, six blank samples were analyzed to check if there 
were any interfering peaks in the chromatogram that could potentially complicate the analysis.

\subsection{Relative Recovery and Precision}

Relative recovery was determined using the following equation:

$$
\% \text { Relative recovery }=100 \times\left(\mathrm{C}_{\text {found }}-\mathrm{C}_{\text {real }}\right) / \mathrm{C}_{\text {added }}
$$

Where, $\mathrm{C}_{\text {found }}$ and $\mathrm{C}_{\mathrm{added}}$ are the concentration of the concerning PAH in the real/blank sample after and before the addition of known amounts of the working solution, respectively. Meanwhile, precision (RSD\%) of the method was investigated on three replicate experiments without any internal standard.

\subsection{Linearity and Evaluation of $L O Q$}

Linearity of the method was tested by spiking the blank samples at ten concentration levels over the range of 2.0$100.0 \mathrm{ng} / \mathrm{g}$ for the test PAHs. Calibration curves were constructed by plotting the PAH signal obtained, which was the average of three measurements against the respective concentration of the test PAH. The LOQs were determined based on the following definition: The lowest concentration at which the error falls between $-20 \%$ and $+20 \%$ with the maximum RSD of $20 \%$ obtained under three measurements.

\section{Results and Discussion}

\subsection{Optimization of Ultrasound Assisted QuEChERS}

To find the optimum conditions for the test PAHs extraction in the soil samples, initially sonication time was tested in the range of 1-5 min. The result revealed that the recovery of the PAHs slightly enhanced, whilst the ultrasonic treatment was increased from 1 to $4 \mathrm{~min}$. However, the recovery remained almost unchanged beyond $4 \mathrm{~min}$ (date not shown). Therefore, 4 min was taken as the optimum sonication time. Further on, the variation in the volume ratio of ACN:Water was made in such a way that the extracted upper layer after the ultrasound assisted QuEChERS treatment was $2 \mathrm{ml}$ right before the cleanup process. Accordingly, the ratio of ACN:Water 5:2 ml was applied for the initial extraction of the PAHs from the soils. Other factors such as the amount of sample $(2.0 \mathrm{~g})$, the sonication parameters $(50 \mathrm{KHz}, 110 \mathrm{~W})$, and the mix salt amounts $\left(\mathrm{MgSO}_{4}: \mathrm{NaCl} ; 4: 1 \mathrm{~g}\right)$ and the clean-up materials (50, 50 and $150 \mathrm{mg}$ of $\mathrm{C}_{18}$, PSA and anh. $\mathrm{MgSO}_{4}$ ) were maintained invariable due to being reported in the literature $[7,19]$.

\subsection{Optimization of the Influential Parameters in DLLME}

To the clear supernatant $(\sim 1 \mathrm{ml}$ of $\mathrm{ACN})$ obtained from the clean-up process, different amounts of $\mathrm{C}_{2} \mathrm{Cl}_{4}, \mathrm{CCl}_{4}, \mathrm{CHCl}_{3}$ were added in order to find out the optimum conditions. It should be noted that the sedimented phase, resulting from the three above-mentioned solvents later must fall in the range of $5 \pm 0.5 \mu \mathrm{l}$. Accordingly, the optimal conditions of DLLME was found out to be $12 \mu \mathrm{l}$ of $\mathrm{C}_{2} \mathrm{Cl}_{4}$ as reported elsewhere [16] (see Figure 1,2). It is noted that the PAHs recovery from the spiked soil samples at $10 \mathrm{ng} / \mathrm{g}$ level was taken as the response of the optimization experiments.

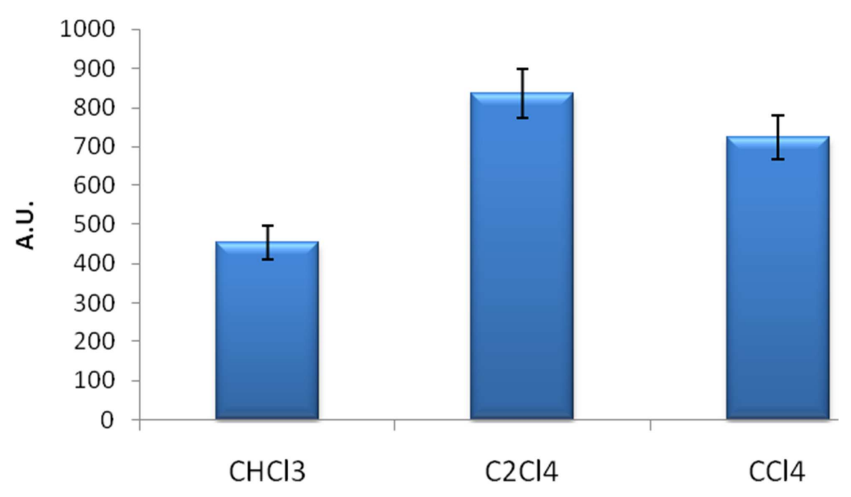

Figure 1. The effect of type of extraction solvent used in DLLME procedure.

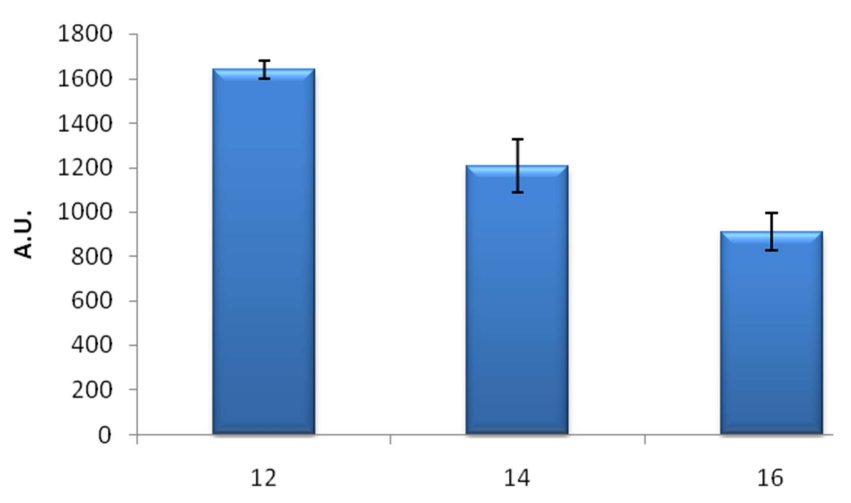

Figure 2. The effect of volume of $\mathrm{C}_{2} \mathrm{Cl}_{4}(\mu \mathrm{l})$ on the extraction efficiency of the method.

\subsection{Results Regarding the Analytical Parameters}

According to the results obtained, there were no interfering peaks, originating from the blank sample matrix or the chemicals and reagents used, at the same retention time of the test PAHs in any of the six blank samples studied in the selectivity experiments (Figure $3 \mathrm{~B}$ ).

The results also demonstrated a fair linearity for the test PAHs within the mentioned-above test range with the minimum correlation coefficient of 0.98 . The LOQs were found to be in the range of $2.5-4.0 \mathrm{ng} / \mathrm{g}$ based on the following definition: The lowest concentration at which the error falls between $-20 \%$ and $+20 \%$ with the maximum RSD of $20 \%$ obtained under three measurements (see Table 1 ).

Table 1. Analytical data of the method.

\begin{tabular}{llc}
\hline PAHs & SIM & LOQ (ng/g) \\
\hline Acenaphthylene & 152 & 4.0 \\
Acenaphthene & 153 & 3.5 \\
Fluorene & 166 & 2.5 \\
phenantherene & 178 & 2.0 \\
Anthracene & 178 & 2.0 \\
Fluoranthene & 202 & 2.0 \\
Pyrene & 202 & 2.5 \\
Chrysene & 228 & 3.0 \\
\hline
\end{tabular}




\subsection{Analysis of Real Samples}

Application of ultra-assisted QuEChERS along with DLLME to the soil samples from three different soil samples was assessed. Of the three suspected samples analyzed, none were found to be contaminated with the test PAHs. The method was successfully applied to the analysis of the test PAHs in the above-mentioned samples spiked at $5.0 \mathrm{ng} / \mathrm{g}$ level to investigate the extraction efficiency of the method.

The RSD\% results based on three similar measurements were within the range of $4.8-15.9 \%$, as presented in Table 2. The Table's data also demonstrated good relative recoveries in the range of $81-92 \%$, indicating that the applied ultrasound QuEChERS combined with DLLME is highly efficient for the measurement of the test PAHs in various soil samples. The GC/MS chromatograms relating to A) the standard solution sample and B) the blank sample are shown in Figure3. As can be seen and deducted, the chromatograms are almost free of matrix effects.

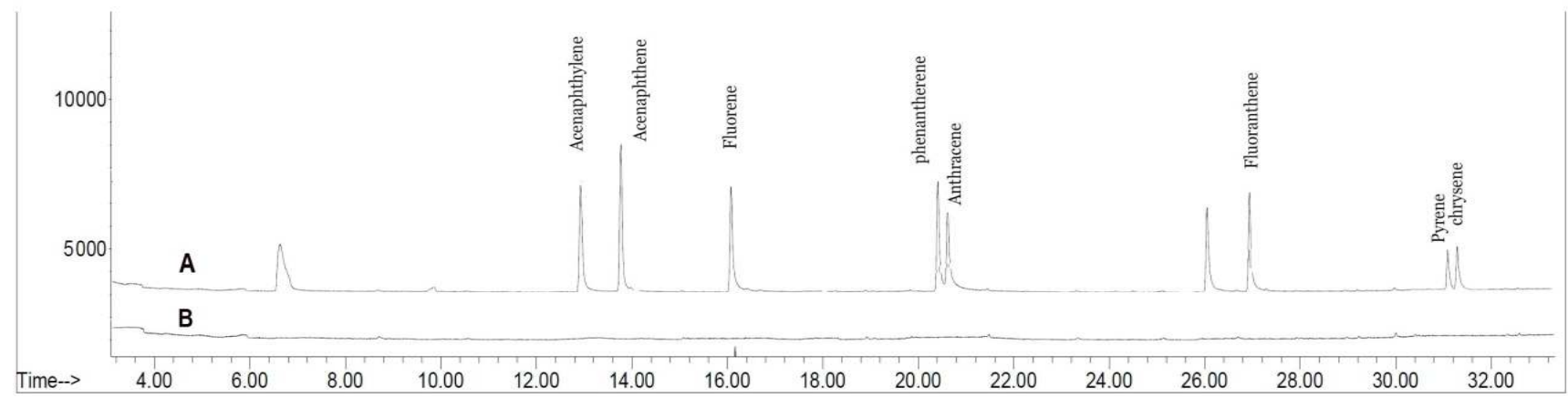

Figure 3. The GC/MS chromatograms relating to A) the standard solution and B) the blank sample.

Table 2. Analysis of real samples.

\begin{tabular}{|c|c|c|c|c|c|c|c|c|c|}
\hline \multirow{2}{*}{ PAHs } & \multicolumn{3}{|l|}{1} & \multicolumn{3}{|l|}{2} & \multicolumn{3}{|l|}{3} \\
\hline & ${ }^{a} \mathbf{R C}$ & Found $^{\mathrm{b}}$ & RR (\%) & RC & Found & RR (\%) & $\mathrm{RC}$ & Found & RR (\%) \\
\hline Acenaphthylene & ${ }^{c} \mathrm{ND}$ & $4.0 \pm 0.6$ & 81 & ND & $4.3 \pm 0.6$ & 86 & ND & $4.3 \pm 0.4$ & 85 \\
\hline Acenaphthene & ND & $4.2 \pm 0.5$ & 84 & ND & $4.0 \pm 0.4$ & 81 & ND & $4.2 \pm 0.6$ & 84 \\
\hline Fluorene & ND & $4.6 \pm 0.4$ & 92 & ND & $4.0 \pm 0.3$ & 81 & ND & $4.1 \pm 0.5$ & 82 \\
\hline Phenenthrene & ND & $4.4 \pm 0.7$ & 85 & ND & $4.2 \pm 0.5$ & 83 & ND & $4.6 \pm 0.6$ & 91 \\
\hline Anthracene & ND & $4.6 \pm 0.5$ & 91 & ND & $4.1 \pm 0.6$ & 82 & ND & $4.6 \pm 0.5$ & 92 \\
\hline Fluoranthene & ND & $4.1 \pm 0.2$ & 82 & ND & $4.2 \pm 0.6$ & 84 & ND & $4.5 \pm 0.5$ & 90 \\
\hline Pyrene & ND & $4.2 \pm 0.6$ & 83 & ND & $4.6 \pm 0.5$ & 91 & ND & $4.3 \pm 0.6$ & 86 \\
\hline Chrysene & ND & $4.4 \pm 0.5$ & 88 & ND & $4.5 \pm 0.4$ & 89 & ND & $4.6 \pm 0.4$ & 92 \\
\hline
\end{tabular}

a: real concentration; b: mean $\pm \mathrm{sd}(\mathrm{n}=3)$; : non-detected

\section{Conclusion}

The present study centers on a novel modified method in which the initial extraction of selected PAHs is carried out by ultrasound assisted QuEChERS treatment followed by a further preconcentration by DLLME procedure. Several influential parameters including ultrasound extraction time, initial extraction and disperser solvent and their respective volumes were all evaluated to achieve the optimal conditions. Afterwards, the method was successfully applied to the analysis of the test PAHs in real soil samples. Finally, the method seems to be simple, low cost and highly efficient for the analysis of PAHs in soil samples.

\section{References}

[1] L. Duedahl-Olesen, Polycyclic aromatic hydrocarbons (PAHs) in foods, National Food Institute Technical University of Denmark, Denmark, Woodhead Publishing Limited: 2013.

[2] R. Dabestani and I. N. Ivanov "A Compilation of Physical, Spectroscopic and Photophysical Properties of Polycyclic
Aromatic Hydrocarbons" Photochemistry and Photobiology, vol. 70, pp. 10-34, 1999.

[3] T. Vo-Dinh, J. Fetzer and A. D. Campiglia "Monitoring and characterization of polyaromatic compounds in the environment" Talanta, vol. 47, pp. 943-969, 1998.

[4] P. Villar, M. Callejón, E.Alonso, J. C. Jiménez and A. Guiraúm"Optimization and validation of a new method of analysis for polycyclic aromatic hydrocarbons in sewage sludge by liquid chromatography after microwave assisted extraction" Analytica Chimica Acta, vol. 524, pp. 295-304, 2004.

[5] L. Correia-Sá, V. C. Fernandes, M. Carvalho, C. Calhau, V. F. Domingues and C. Delerue-Matos "Optimization of QuEChERS method for the analysis of organochlorine pesticides in soils with diverse organic matter" Journal of Separation Science, vol. 35, pp. 1521-1530, 2012.

[6] C. G. Pinto, S. H. Martín, J. L. P. Pavón and B. M. Cordero (2011) "A simplified Quick, Easy, Cheap, Effective, Rugged and Safe approach for the determination of trihalomethanes and benzene, toluene, ethylbenzene and xylenes in soil matrices by fast gas chromatography with mass spectrometry detection" Analytica Chimica Acta, vol. 689, pp. 129-136, 2011. 
[7] V. Jose, L. Correia-Sá, P. Paíga, I. Bragança, V. C. Fernandes, V. F. Domingues and C. Delerue-Matos "QuEChERS and soil analysis" An Overview. Sample preparation, pp. 54-77, 2013.

[8] S. Kahn, L. Aijun, S. Zhang, Q. Hu and Y. G.Zhu, "Accumulation of polycyclic aromatic hydrocarbons and heavy metals in lettuce grown in the soils contaminated with long-term wastewater irrigation" Journal of Hazardous Materials, vol.152, pp. 506-515, 2008.

[9] M. Nording, K. Frech, Y. Persson, M. Forsman and P. Haglund, "Accumulation of polycyclic aromatic hydrocarbons and heavy metals in lettuce grown in the soils contaminated with long-term wastewater irrigation”Analytica Chimica Acta, vol. 555, pp. 107-113, 2006.

[10] T. Wenzl, R. Simon, J. Kleiner and E. Anklam, ”Analytical methods for polycyclic aromatic hydrocarbons (PAHs) in food and the environment needed for new food legislation in the European Union, Trends in Analytical Chemistry, vol. 25, pp. 716-725, 2006.

[11] S. S. Caldas, C. M. Bolzan, M. B. Cerqueira, D. Tomasini, E. B. Furlong, C. Fagundes and E. G. Primel, "Evaluation of a Modified QuEChERS Extraction of Multiple Classes of Pesticides from a Rice Paddy Soil by LC-APCI-MS/MS" Journal of Agricultural Food Chemistry, vol. 59, pp. 1191811926, 2011.

[12] A. Rashid, S. Nawaz, H. Barker, I. Ahmad and M. Ashraf, "Development of a simple extraction and clean-up procedure for determination of organochlorine pesticides in soil using gas chromatography-tandem mass spectrometry" Journal of Chromatography A, vol. 1217, pp. 2933-2939, 2010.

[13] Y. H. Wang, L. W. Du, X.M. Zhou, H.H. Tan, L.Y. Bai, D.Q.
Zeng and H. Tian, "QuEChERS extraction for high performance liquid chromatographic determination of pyrazosulfuron-ethyl in soils" Journal of Chemical Society of Pakistan, vol. 34, pp.28-32, 2012.

[14] C. G. Pinto, M. E. F. Laespada, S. H. Martín, A. M. C. Ferreira, J. L. P. Pavón and B. M. Cordero "Simplified QuEChERS approach for the extraction of chlorinated compounds from soil samples" Talanta, vol. 81, pp. 385-391, 2010 .

[15] M. Asensio-Ramos, J. Hernandez-Borges, L. M. Ravelo-Perez and M. A. Rodriguez-Delgado "Evaluation of a modified QuEChERS method for the extraction of pesticides from agricultural, ornamental and forestal soils" Analytical and Bioanalytical Chemistry, vol. 396, pp. 2307-2319, 2010.

[16] M. Rezaee, Y. Assadi, M. R. Milani-Hosseini, E. Aghaee, F. Ahmadi and S. Berijani "Determination of organic compounds in water using dispersive liquid-liquid microextraction" Journal of Chromatography A, vol. 1116, pp. 1-9, 2006.

[17] M. Rezaee, Y. Yamini, M. Moradi, A. Saleh, M. Faraji and M. H. Naeen "Supercritical fluid extraction combined with dispersive liquid-liquid microextraction as a sensitive and efficient sample preparation method for determination of organic compounds in solid samples" Journal of Supercritical Fluids, vol. 55, pp.161-168, 2010.

[18] M. Rezaee, Y. Yamini and M. Faraji, "Evolution of dispersive liquid-liquid microextraction method" Journal of Chromatography A, vol. 1217, pp. 2342-2357, 2010.

[19] J. Kowalski, R. A. Amanda and J. Cochran (2013) "QuEChERS: The Concept"www.sepscience.com; Europe, 5. 